\title{
Multiple outer-shell ionization effect in inner-shell x-ray production by light ions
}

\author{
G. Lapicki and R. Mehta \\ Department of Physics, East Carolina University, Greenville, North Carolina 27858 \\ J. L. Duggan, P. M. Kocur, J. L. Price, and F. D. McDaniel \\ Department of Physics, North Texas State University, Denton, Texas 76203
}

(Received 24 October 1985)

\begin{abstract}
$L$-shell $\mathrm{x}$-ray production cross sections by $0.25-2.5-\mathrm{MeV}{ }_{2}^{4} \mathrm{He}^{+}$ions in ${ }_{28} \mathrm{Ni},{ }_{29} \mathrm{Cu},{ }_{32} \mathrm{Ge},{ }_{33} \mathrm{As}$, ${ }_{37} \mathrm{Rb},{ }_{38} \mathrm{Sr},{ }_{39} \mathrm{Y},{ }_{40} \mathrm{Zr}$, and ${ }_{46} \mathrm{Pd}$ are reported. The data are compared to the first-Born approximation and the ECPSSR theory that accounts for the projectile energy loss (E) and Coulomb deflection (C) as well as the perturbed-stationary-state (PSS) and relativistic (R) effects in the treatment of the target $L$-shell electron. Surprisingly, the first Born approximation appears to converge to the data while the ECPSSR predictions underestimate them in the low-velocity limit. This is explained as the result of improper use of single-hole fluorescence yields. A heuristic formula is proposed to account for multiple ionizations in terms of a classical probability for these phenomena and, after it is applied, the ECPSSR theory of $L$-shell ionization is found to be in good agreement with the data.
\end{abstract}

\section{INTRODUCTION}

Multiple ionizations are known to affect inner-shell fluorescence yields. ${ }^{1}$ The standard fluorescence yieldscalculated for atoms with a single vacancy in their inner shells-may increase significantly when there is an appreciable probability that outer shells are also ionized. A bewildering complexity of possible transitions in multiply ionized atoms, foils all-inclusive attempts to modify the standard fluorescence yields for the maze of such transitions. Even if a rigorous formula was to be derived to cover all possibilities, its utility would be marred by imprecise knowledge of the degrees to which various outer shells had simultaneously undergone ionization in a collision. We propose instead, in the Appendix, a formula that modifies these yields in an approximate manner and relies on an easily scalable and classical expression for the probability of outer-shell ionization. This probability is proportional to $\left(Z_{1} / v_{1}\right)^{2}$, the square of the projectile charge-to-velocity ratio, and-except for $Z_{1}=1$ ions-its validity is limited to the $Z_{1} / v_{1} \leqq \sqrt{2}$ projectiles. To study experimentally the effect of multiple ionization under such a restriction and yet to have $\left(Z_{1} / v_{1}\right)^{2}$ as large as possible, we chose $0.25-2.5-\mathrm{MeV}{ }_{2}^{4} \mathrm{He}$ ions as projectiles so that $1.26 \geq Z_{1} / v_{1} \geq 0.40$. Also, to maximize this effect, we have selected $L$ shells in relatively light target atoms $\left(28 \leq Z_{2} \leq 46\right)$ for which the standard fluorescence yields are small, i.e., in the 0.009-0.05 range.

Section II contains a short survey of $L$-shell x-ray production by helium ions in the elements that we consider light-with respect to heavier atoms for which such data are usually reported-and yet of a sufficiently large atomic number so that their $L$ shells can be treated as inner shells. Our experiment for such collisions is briefly described in Sec. III and its results as well as the data of others are compared with theory in Sec. IV. In the concluding Sec. IV B, our method of accounting for multiple ionizations of outer shells is tested against the semiempirically deduced mean fluorescence yields in the $L$-shell ionization.

\section{SURVEY OF L-SHELL X-RAY PRODUCTION BY HELIUM IONS IN LIGHT TARGET ATOMS}

A search of the literature shows that most of the published results for $L$-shell ionization cross sections are for heavy elements whose $L$-shell binding energies are significantly greater than $3 \mathrm{keV}$, i.e., for target atoms with the atomic number $Z_{2}>46$. The measurements in this work are for the $L$-shell $\mathrm{x}$ rays whose energies are below $3 \mathrm{keV}$. Experimental difficulties encountered in making such measurements include (a) large experimental uncertainties in the detector efficiency below $3 \mathrm{keV}$, (b) contaminant $\mathrm{x}$ rays from light $K$-shell elements that exist as impurities on the carbon backings, and (c) for the target elements with the atomic number $Z_{2}<47$, the $L$-subshell transitions are spaced closely in energy, making it difficult to resolve individual subshell transitions and hence extract cross sections.

$L$-shell x-ray production and ionization cross sections by helium ions were tabulated by Hardt and Watson ${ }^{2}$ and by Gray $^{3}$ (with publications until 1977). In the $28 \leq Z_{2} \leq 46$ range, these surveys include only the ${ }_{34} \mathrm{Se}$ and ${ }_{42} \mathrm{Mo}$ measurements of $\mathrm{Komarek}^{4}$ who used $5.3-\mathrm{MeV} \alpha$ particles from a ${ }^{210} \mathrm{Po}$ decay. He was able to produce a range of projectile energies after penetration of these $\alpha$ particles through Mylar foils of predetermined thicknesses. However, the slowest $\alpha$ particles at $2.9 \mathrm{MeV}$ are above our projectile energy range. References 2 and 3 do not list the 1971 data of Shima et al. ${ }^{5}$ on ${ }_{29} \mathrm{Cu}$ and ${ }_{42} \mathrm{Mo}$ by ${ }^{3} \mathrm{He}$ and ${ }^{4} \mathrm{He}$ projectiles that span extremely low energies in the $0.16-0.32-\mathrm{MeV}$ range. Since 1977 additional measurements have been reported by Button et al. ${ }^{6}$ for targets of ${ }_{29} \mathrm{Cu},{ }_{30} \mathrm{Zn},{ }_{31} \mathrm{Ga},{ }_{32} \mathrm{Ge},{ }_{33} \mathrm{As},{ }_{34} \mathrm{Se}$, and ${ }_{35} \mathrm{Br}$ 
with $\mathrm{He}^{+}$ions in the energy range $0.4-2.4 \mathrm{MeV}$; they were compared to the plane-wave Born approximation (PWBA), ${ }^{7}$ binary-encounter approximation (BEA), ${ }^{8}$ and precursors of the ECPSSR theory ${ }^{9}$ for the $L$ shell [ECPSSR stands for the projectile ion's energy loss (E) and Coulomb deflection $(C)$ and for perturbed-stationarystate (PSS) and relativistic (R) effects in the inner shell of the target atom that are accounted for in the theory of inner-shell ionization], i.e., an early approach that accounted for binding and Coulomb deflection effects ${ }^{10}$ and a later CPSSR formulation. ${ }^{11}$ All the theories generally predicted the trend of the data very well; the actual agreement ranged from fair to good, and excellent in some cases. Poncet and Engelmann ${ }^{12}$ compared their $\sigma_{L X}$ measurements by $10-30-\mathrm{MeV} \alpha$ particles on ${ }_{39} \mathrm{Y}$ with the PWBA (Ref. 7) and BEA (Ref. 8). The PWBA was found to be in good agreement with their data for yttrium. For heavier targets $\left(Z_{2} \geq 50\right)$, however, the PWBA calculations were above the $L$-shell data of Ref. 12. Only for gold, the heaviest element in these measurements, the PWBA fell below the data indicating a clear shortcoming of the standard PWBA (Ref. 7) which is based on nonrelativistic $L$-shell wave functions. Ishii et al., ${ }^{13}$ in their $Z_{1}^{2}$-dependence studies, reported ratios of $x$-ray yields of ${ }_{39} \mathrm{Y}$ by protons and ${ }^{3} \mathrm{He}$ ions of the same velocities corresponding to $7,9,12,16$, and $21 \mathrm{MeV} / \mathrm{u}$. The ratio tended to unity with the increased projectile energy, just as the ECPSSR converges to the PWBA results in the highvelocity limit. In a more recent study by Jesus et al. ${ }^{14} \alpha$ particles of lower energy $(0.8-1.8 \mathrm{MeV})$ on ${ }_{32} \mathrm{Ge},{ }_{35} \mathrm{Br}$, ${ }_{40} \mathrm{Zr},{ }_{42} \mathrm{Mo}$, and ${ }_{47} \mathrm{Ag}$ were used. $L$-shell cross sections were measured for ${ }_{40} \mathrm{Zr}$, ${ }_{42} \mathrm{Mo}$, and ${ }_{47} \mathrm{Ag}$ only; germanium and bromine were utilized only to determine ratios of $\sigma_{L X}(\alpha$ particle $) / \sigma_{L X}$ (deuteron) at equal projectile velocities in a test of the binding effect.

In the present work, $L$-shell $x$-ray production cross sections have been measured for $0.25-2.5-\mathrm{MeV}{ }^{4} \mathrm{He}^{+}$ions incident on thin solid targets (thickness in $\mu \mathrm{g} / \mathrm{cm}^{2}$ in parentheses) of ${ }_{28} \mathrm{Ni}(6.4),{ }_{29} \mathrm{Cu}(19),{ }_{32} \mathrm{Ge}(28),{ }_{33} \mathrm{As}$ (12), ${ }_{37} \mathrm{Rb}$ (29), ${ }_{38} \mathrm{Sr}$ (11), ${ }_{39} \mathrm{Y}(20),{ }_{40} \mathrm{Zr}$ (17), and ${ }_{46} \mathrm{~Pb}$ (11).

\section{EXPERIMENTAL PROCEDURE AND DATA ANALYSIS}

Ion beams of ${ }^{4} \mathrm{He}^{+}$were obtained from the 2.5-MV Van de Graaff accelerator at North Texas State University. Thin targets of ${ }_{28} \mathrm{Ni},{ }_{29} \mathrm{Cu},{ }_{32} \mathrm{Ge},{ }_{33} \mathrm{As},{ }_{37} \mathrm{Rb},{ }_{38} \mathrm{Sr},{ }_{39} \mathrm{Y}$, ${ }_{40} \mathrm{Zr}$, and ${ }_{46} \mathrm{Pd}$ were prepared by vacuum evaporation and deposition techniques. The $10-20-\mu \mathrm{g} / \mathrm{cm}^{2}$ carbon foils, used as backing material for these targets, were screened to make sure there was a minimal presence of lowatomic-number contaminants. The elemental layer then deposited on the carbon was thin enough so that the energy loss sustained by the projectile ion in going through was negligible, but at the same time the layer was thick enough to provide $x$-ray peaks that gave good statistics for reasonable running times. Further details of this technique for making contaminant-free targets are given in Ref. 15.

Simultaneous measurement of the yield of the $L$-shell $x$-rays and the yield of the scattered particles during the ion-target interaction was used to determine the $L$-shell $\mathrm{x}$-ray production cross section. The $\mathrm{Si}(\mathrm{Li}) \mathrm{x}$-ray detector and the Si surface-barrier particle detector were positioned at $90^{\circ}$ and $150^{\circ}$ to the incident beam, respectively. The target was positioned at $45^{\circ}$ to the incident beam direction. Particulars of the experimental geometry, data analysis, and the efficiency of the $\mathrm{Si}(\mathrm{Li})$ detector were described elsewhere. ${ }^{16,17}$ The absolute uncertainty in the data reported here ranges from $13 \%$ to $26 \%$, which is primarily due to uncertainty in the (i) background subtraction and polynomial fitting $(2-13 \%)$ and (ii) efficiency of the $\mathrm{Si}(\mathrm{Li})$ detector at the $L$-shell $\mathrm{x}$-ray energies $(9-19 \%)$. The largest uncertainty due to efficiency occurs at low $L$ shell $x$-ray energies for nickel and copper due to the steep-

TABLE I. $L$-shell $\mathrm{x}$-ray production cross sections in barns for ${ }^{4} \mathrm{He}^{+}$ions.

\begin{tabular}{|c|c|c|c|c|c|c|c|c|c|c|c|}
\hline \multirow{2}{*}{$\begin{array}{l}\text { Target } \\
\text { element }\end{array}$} & \multirow[b]{2}{*}{ Source } & \multicolumn{10}{|c|}{ Projectile energy (MeV) } \\
\hline & & 0.25 & 0.50 & 0.75 & 1.0 & 1.25 & 1.50 & 1.75 & 2.00 & 2.25 & 2.50 \\
\hline \multirow[t]{2}{*}{${ }_{28} \mathrm{Ni}$} & Measured & 280 & 1030 & 1340 & 2360 & 3060 & 4370 & 5640 & 6600 & 6880 & 6910 \\
\hline & Theory-ECPSSR & 32.3 & 305 & 908 & 1770 & 2770 & 3820 & 4840 & 5820 & 6720 & 7550 \\
\hline \multirow[t]{2}{*}{${ }_{29} \mathrm{Cu}$} & Measured & 89.7 & 360 & 1130 & 1900 & 2970 & 3950 & 4520 & 5300 & 5710 & \\
\hline & Theory-ECPSSR & 24.8 & 242 & 733 & 1460 & 2330 & 3270 & 4210 & 5120 & 5980 & 6780 \\
\hline \multirow[t]{2}{*}{${ }_{32} \mathrm{Ge}$} & Measured & 24.1 & 110 & 529 & 884 & 1460 & 1900 & 2420 & 2900 & 3430 & 3600 \\
\hline & Theory-ECPSSR & 8.43 & 93.5 & 300 & 631 & 1070 & 1580 & 2130 & 2700 & 3270 & 3830 \\
\hline \multirow[t]{2}{*}{${ }_{33} \mathrm{As}$} & Measured & 19.4 & 118 & 282 & 546 & 899 & 1290 & 1580 & 2060 & 2670 & 2860 \\
\hline & Theory-ECPSSR & 5.84 & 67.6 & 221 & 472 & 809 & 1210 & 1660 & 2130 & 2620 & 3090 \\
\hline \multirow[t]{2}{*}{${ }_{37} \mathbf{R b}$} & Measured & 11.2 & 54.6 & 137 & 234 & 407 & 586 & 762 & 956 & 1270 & 1010 \\
\hline & Theory-ECPSSR & 1.58 & 23.3 & 82.4 & 184 & 330 & 515 & 736 & 984 & 1250 & 1540 \\
\hline \multirow[t]{2}{*}{${ }_{38} \mathrm{Sr}$} & Measured & 8.09 & 33.7 & 91.3 & 156 & 248 & 383 & 559 & 743 & 852 & 1060 \\
\hline & Theory-ECPSSR & 1.12 & 17.7 & 64.0 & 145 & 261 & 412 & 593 & 800 & 1030 & 1270 \\
\hline \multirow[t]{2}{*}{${ }_{39} \mathrm{Y}$} & Measured & 5.76 & 30.0 & 72.5 & 135 & 232 & 335 & 465 & 636 & 729 & 920 \\
\hline & Theory-ECPSSR & 0.796 & 13.5 & 49.9 & 114 & 208 & 330 & 478 & 650 & 840 & 1050 \\
\hline \multirow[t]{2}{*}{${ }_{40} \mathrm{Zr}$} & Measured & 2.61 & 22.6 & & & & & & 708 & 915 & 893 \\
\hline & Theory-ECPSSR & 0.585 & 10.6 & 40.3 & 93.3 & 171 & 273 & 398 & 545 & 708 & 887 \\
\hline \multirow[t]{2}{*}{${ }_{46} \mathrm{Pd}$} & Measured & 0.462 & 6.20 & 21.4 & 41.0 & 63.0 & 106 & 132 & 181 & 190 & 258 \\
\hline & Theory-ECPSSR & 0.085 & 2.56 & 11.4 & 28.8 & 55.4 & 91.7 & 138 & 193 & 258 & 331 \\
\hline
\end{tabular}


ness of the fitted curve; the accuracy of the efficiency curve for other elements may be seriously affected by $x$ ray absorption edges.

\section{COMPARISON WITH IONIZATION THEORIES}

A. Using the standard fluorescence yield

The two main process responsible for inner-shell ionization due to charged-particle excitation are direct ionization to the target continuum (DI) and the target electron capture by the projectile ion (EC). DI plus EC can be predicted by the first Born [PWBA for DI (Ref. 7) and Oppenheimer-Brinkman-Kramers calculation by Nikelaev (OBKN of Ref. 18) for EC] and by the ECPSSR (Ref. 9) approach. The ECPSSR goes beyond first Born by including the effects of energy loss, Coulomb deflection, and the relativistic effects, in the perturbed-stationarystate theory.

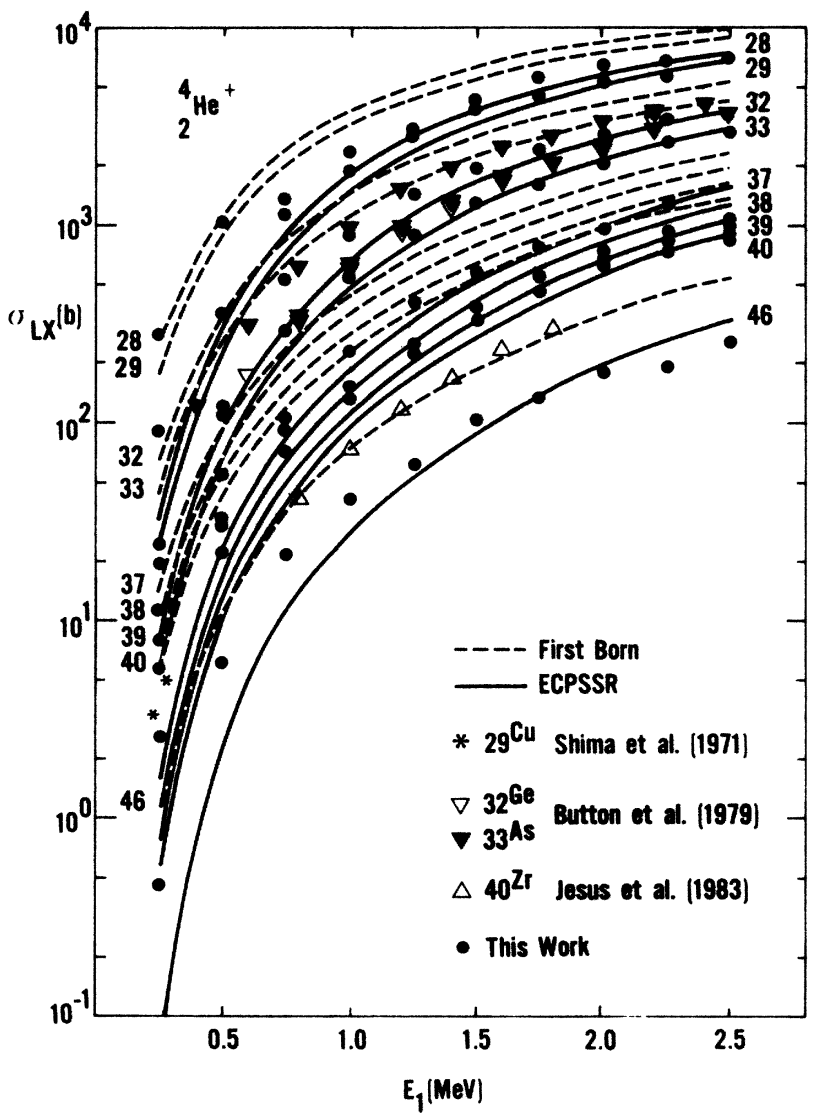

FIG. 1. $L$-shell $\mathrm{x}$-ray production cross sections in nickel, copper, germanium, arsenic, rubidium, strontium, yttrium zirconium, and palladium as a function of the $\mathrm{He}^{+}$-ion energy. The data of this work can be recognized with the aid of Table I. Predictions of the first Born approximation from Refs. 7 and 18 (the dashed curves identified in the left of the figure by the atomic numbers of corresponding elements), results of the ECPSSR theory from Ref. 9 (the solid curves marked by the atomic numbers in the right), and the data from Refs. 5, 6, and 14 are shown for comparison. As both calculations indicate, the measured cross sections decrease with the increasing atomic number of the target element and with the decreasing energy of the projectile; these calculated and experimental trends allow for an unambiguous reading of this figure.
Table I lists both the measured and the ECPSSR values of the $L$-shell $x$-ray production cross section for incident ${ }^{4} \mathrm{He}^{+}$ions. The absolute errors for the cross-section measurements range from $13 \%$ to $26 \%$ with the larger errors at lower energies and for lower- $Z_{2}$ targets. The $L$-shell ionization cross sections calculated in the ECPSSR approximation were converted to the production cross sections using the standard single-hole fluorescence yields and Coster-Kronig transition rates from Krause. ${ }^{19}$

Figure 1 displays our total $L$-shell $x$-ray production cross section data $\sigma_{L X}$ versus the energy of helium ions. $L$-shell $X$-ray production cross sections predicted by the first Born and the ECPSSR theories are shown as dashed and solid curves, respectively. Also shown are the measurements of Refs. 5, 6, and 14. The present data follow the gross trend of the theories with increasing energy of the projectile. Uncharacteristically, the first Born calculation overestimates the data at high energies and appears to converge to them at the lowest energies. The cross sections of Button et al. ${ }^{6}$ as well as our data are generally in good agreement with the ECPSSR theory; the ${ }_{40} \mathrm{Zr}$ solid curve is about midway between our data and those of Ref. 14. However, our measurements at $0.25 \mathrm{MeV}$ are factors of from $\sim 3$ to $\sim 9$ above the ECPSSR predictions.

This becomes clear in Fig. 2 which depicts $\sigma_{L X}$ versus

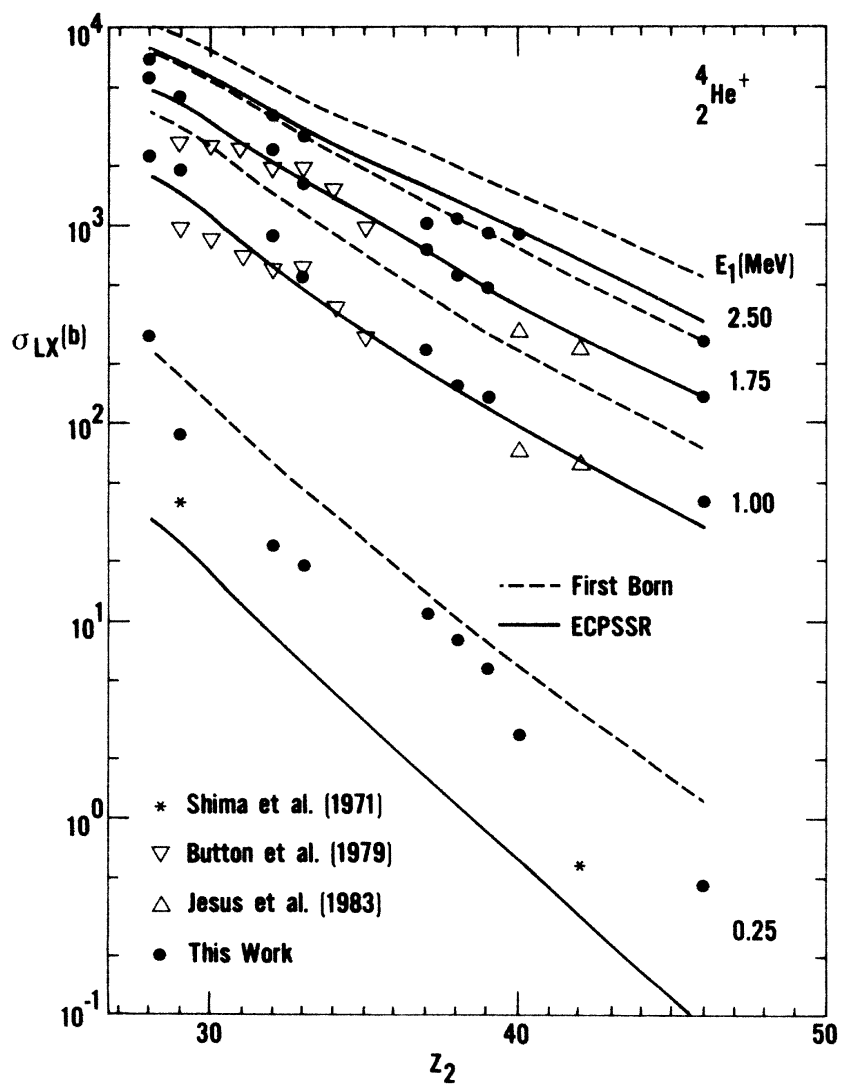

FIG. 2. $L$-shell $\mathrm{x}$-ray production cross sections for ${ }^{4} \mathrm{He}^{+}$ions at energies of $0.25,1.0,1.75$ and $2.5 \mathrm{MeV}$ incident on various targets of atomic numbers $28 \leq Z_{2} \leq 46$. Data, some of which are taken from Refs. 5, 6, and 14, are compared to the first Born approximation and ECPSSR theory. 
target atomic number $Z_{2}$ at four equidistant energies of ${ }_{2}^{4} \mathrm{He}^{+}$. The predictions of ECPSSR are in excellent agreement with present measured points except at $0.25 \mathrm{MeV}$, where ECPSSR grossly underpredicts our measurements and, to a lesser degree, the data of Shima et al. ${ }^{5}$ Measurements of Button et al. ${ }^{6}$ and Jesus et al. ${ }^{14}$ are in good agreement with our data and the ECPSSR predictions for $Z_{2}>30$.

To expose this systematic discrepancy between the data and the ECPSSR theory, we have plotted in Fig. 3 the ratios of experimental cross sections to the cross sections calculated according to this theory. Our data are shown by open, half-closed, and closed circles for the lightest, medium, and heaviest target atoms in search for a possible $Z_{2}$ dependence. The discrepancy appears to be $Z_{2}$ independent, although it may not be purely fortuitous that the largest discrepancies are for ${ }_{28} \mathrm{Ni}$. At all energies our data tend to be above the measurements of Refs. 5, 6, and 14. The low-energy data of Shima et al. ${ }^{5}$ are distinctly below ours; they were obtained, however, in thick-target experiments. One can speculate that if corrections for finite target thickness were made-as suggested, for example, in Refs. 20 and $21-$ cross sections of Ref. 5 would indeed be underestimated by the ECPSSR theory to a larger degree; in effect, ${ }^{21}$ the correction for the finite target thickness amounts to comparison of the observed data with the theory at lower energy than the incident energy, i.e., where the theoretical cross sections are smaller and, henceforth, the ratios for the Shima et al. ${ }^{5}$ data could indeed be larger than shown in Fig. 3.

If one were to stop the discussion here, one would have to conclude that the ECPSSR theory clearly fails at low velocities by increasingly larger factors with the decreasing velocity of the projectile. These factors are as much as fivefold times larger than one finds ${ }^{22}$ in analyses of $K$ shell ionization data; moreover, whereas the $K$-shell data are somewhat overestimated by the ECPSSR theory at low velocities, the $L$-shell data appear to be grossly $u n$ derestimated by this theory.

\section{B. Using the fluorescence yield corrected for multiple ionizations}

We attribute the glaring discrepancies in Fig. 3 between experimental $x$-ray production cross sections and the theoretical values to the conversion of the ECPSSR ionization cross sections that relies on single-hole fluoresence yields. Lack of electrons in multiply ionized atoms inhibits both radiative and nonradiative transitions. X-ray rates are less curtailed than rates for Auger processes which, rather than just one electron needed for an x-ray decay, require two electrons. Hence multiple ionizations enhance the fluorescence yield. A proper correction of this yield demands detailed knowledge of all possible transitions that fill an inner-shell vacancy and the probabilities for ionization of the outer shells that are involved in these transitions. To simplify the myriad of such transitions and ways of their modification, we propose to clump outer shells into a collective state and introduce a classical probability for its ionization. As shown by the formula obtained in the Appendix, multiple ionizations increase the effective fluorescence yield in a dramatic fashion with

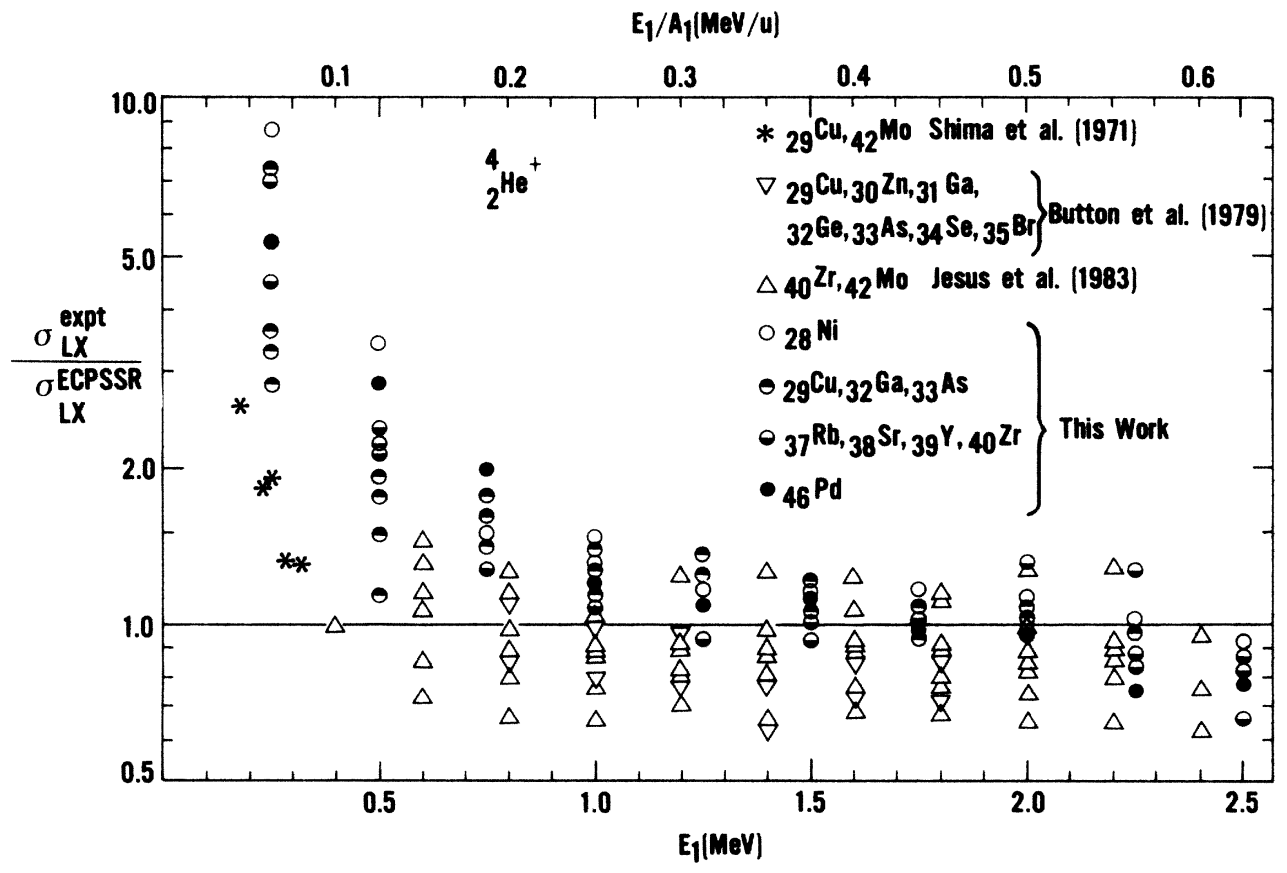

FIG. 3. Ratios $\sigma_{L X}^{\text {expt }} / \sigma_{L X}^{\mathrm{ECPSSR}}$ of the measured $L$-shell $\mathrm{x}$-ray production cross section to the prediction of the ECPSSR theory vs energy $E_{1}$ and energy per unit mass $E_{1} / A_{1}$ of ${ }^{4} \mathrm{He}^{+}$ions. The ECPSSR ionization cross sections were converted to x-ray production cross sections using the single-hole fluorescence yields and Coster-Kronig transition rates of Ref. 19. 


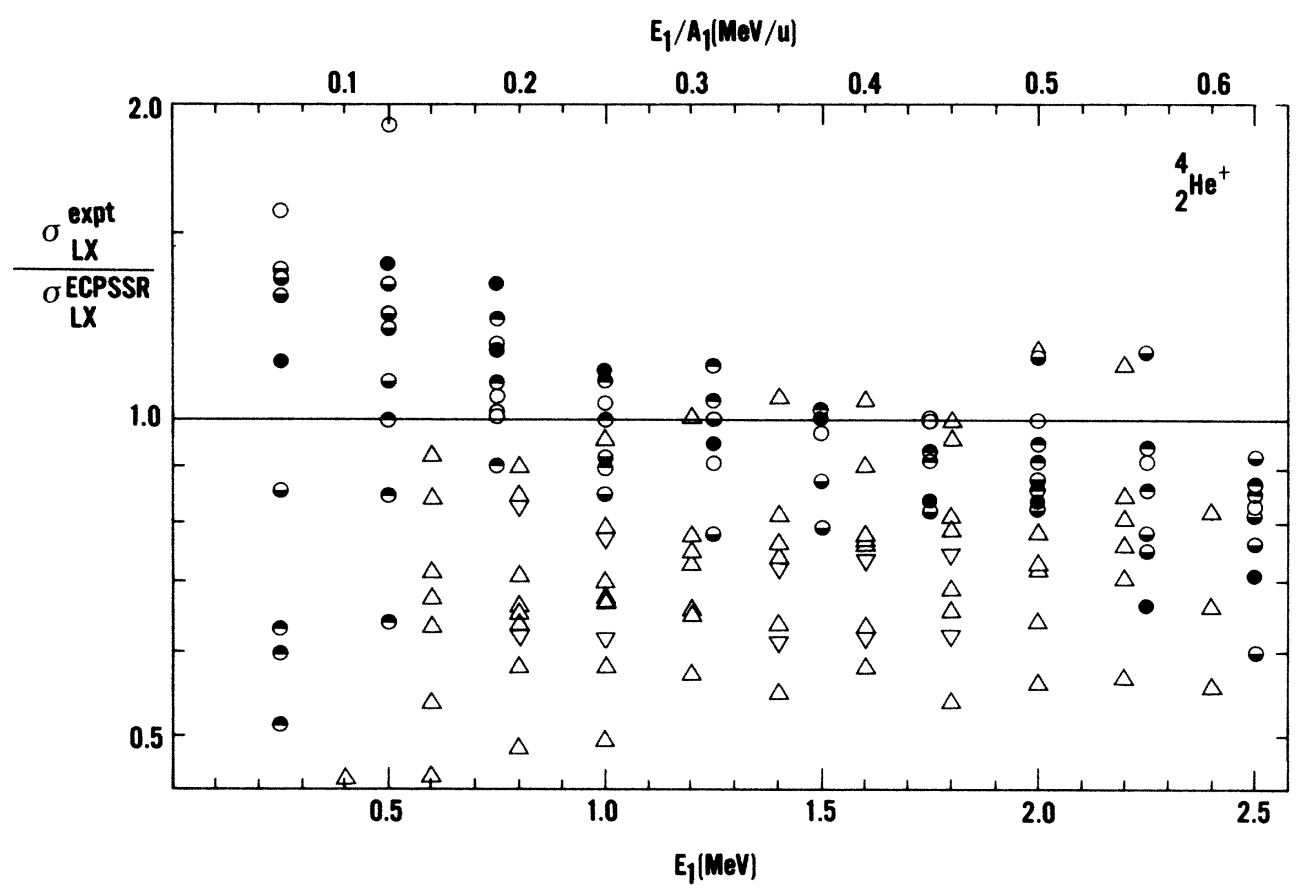

FIG. 4. As Fig. 3 but using the effective fluorescence yield $\bar{\omega}_{L}$ that is modified for multiple ionizations according to Eq. (A3) with $\beta=0.87$. Note that the ordinate has been expanded by a factor of 2 .

the decreasing velocity of the projectile-the conversion of the ECPSSR ionization cross sections would result in larger $\mathrm{x}$-ray production cross sections if larger fluorescence yields were to be employed. Figure 4-note a double magnification of its ordinate in relation to Fig. 3demonstrates that in fact a good agreement between the ECPSSR theory and the data results once the ionization calculations are multiplied by the effective fluorescence yield of Eq. (A3). We have treated $\beta$ in this equation as an adjustable parameter and found that $\beta=0.87$ gives the best least-squares fit to all the data. This value is in excellent agreement with $\beta=0.9$ that is deduced in the Appendix.

To test the $Z_{1}^{2}$ scaling of this equation, we consider the experimental ratios of $\sigma_{L X}\left(\alpha\right.$ particle) to $\sigma_{L X}$ (deuteron) at identical projectile velocities. Such ratios were measured by Jesus et al. ${ }^{14}$ to verify the correctness of the way in which the binding effect has been accounted for in the ECPSSR theory. The ratios of this kind offer a very accurate measure of the binding effect (or the PSS effect that also considers the polarization of the target shell) since they are determined with errors less than $5 \%$, and because other (non-PSS) effects in the ECPSSR theory are essentially canceled out in such ratios. As shown in Fig. 5, contrary to the first Born approximation (dashed line) that predicts $\sigma_{L X}\left(\alpha\right.$ particle) $/ 4 \sigma_{L X}$ (deuteron) $=1$, the ECPSSR (dot-dashed curve) reproduces the trends observed in the experiment. However, the magnified view offered by experiment appears to suggest-as concluded in Ref. 14-that the theory overestimates the role of the binding effect. ${ }^{23}$ Similar findings were obtained in studies of such ratios for $K$-shell ionization ${ }^{24}$ and most recently reaffirmed for the $L$ shell by Jesus et al. ${ }^{25}$ with the projectiles as in Ref. 14 but on ${ }_{79} \mathrm{Au},{ }_{82} \mathrm{Pt}$, and ${ }_{92} \mathrm{U}$. The solid curves are drawn in Fig. 5 after the ECPSSR ionization

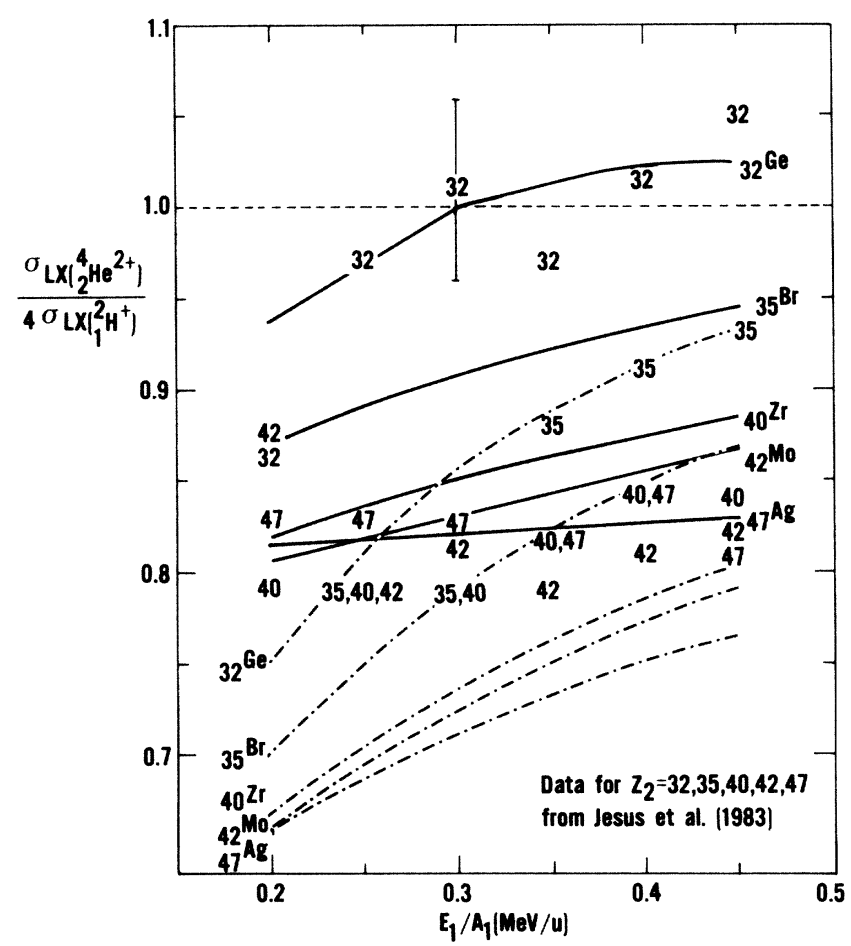

FIG. 5. Ratios of $\sigma_{L X}(\alpha$ particle $) / 4 \sigma_{L X}($ deuteron $)$ at equal projectile velocity. Data are from Ref. 14; they are represented by the atomic number of the target. The dashed line at 1 is the result of the first Born approximation. The dash-dotted curves follow according to the ECPSSR theory of Ref. 9 and singlehole fluorescence yields that are canceled out in these ratios; the target atoms to which they correspond are identified on the left. The solid curves are obtained when the fluorescence yields for $\alpha$ particles and deuterons are different because of multipleionization effect in accordance with Eq. (A3); the target elements to which they correspond are identified on the right. 
calculations for $\alpha$ particles and deuterons are multiplied by $\mathrm{Eq}$. (A3) rather than the same single-hole fluorescence yields.

The agreement with the data is now excellent; the $\sigma_{L X}\left({ }_{2}^{4} \mathrm{He}^{2+}\right) / 4 \sigma_{L X}\left({ }_{1}^{2} \mathrm{H}^{+}\right)$ratios of Fig. 6 in Ref. 25 for heavy targets, where electron capture is negligible (less than $0.01 \%$ ), are also found to be in good agreement with the ECPSSR prescription after the account for multiple ionizations. ${ }^{26}$ This rehabilitates the ECPSSR theory and also confirms, in a quantitative manner, that our method of accounting for multiple ionization is accurate in its prediction of the dependence of this effect on the projectile's velocity as well as on its charge. The central idea of this work is that the fluorescence yield, a link between ionization and $x$-ray cross sections, was at fault. Given any ionization theory and x-ray data, the comparison of the theory to the experimental $x$-ray cross sections hinges on the knowledge of the fluorescence yield. It is this link that we have attempted to repair to make this comparison as proper as possible.

\section{ACKNOWLEDGMENT}

North Texas State University work was supported in part by the Robert A. Welch Foundation and the State of Texas Organized Research Fund.

\section{APPENDIX: A FORMULA FOR THE FLUORESCENCE YIELD CORRECTION DUE TO MULTIPLE IONIZATIONS}

At the outset of our derivation of a formula that accounts for multiple ionization, we make a simplifying assumption: all subshells which are outer with respect to a given shell $S$ undergo ionization with the identical probability $P$ per electron. Under this assumption, the multiple-ionization process is viewed as a removal of one electron from a manifold of outer states ${ }^{27}$ so that the width of all radiative transitions $\Gamma_{S X}^{0}$ into the $S$ shell is narrowed by the same factor to $\Gamma_{S X}^{0}(1-P)$; concurrently, all Auger transition widths $\Gamma_{S A}^{0}$ are decreased to $\Gamma_{S A}^{0}(1-P)(1-P)$ since the nonradiative transitions involve two electrons from outer shells. In this model, the single-hole fluorescence yield $\omega_{S}^{0} \equiv \Gamma_{S X}^{0} /\left(\Gamma_{S X}^{0}+\Gamma_{S A}^{0}\right)$ relates to $\omega_{S}$, the fluorescence yield corrected for multiple ionizations as

$$
\begin{aligned}
\omega_{S} & =\Gamma_{S X}^{0}(1-P) /\left[\Gamma_{S X}^{0}(1-P)+\Gamma_{S A}^{0}(1-P)(1-P)\right] \\
& =\omega_{S}^{0} /\left[1-P\left(1-\omega_{S}^{0}\right)\right] .
\end{aligned}
$$

This formula guarantees that, with $0 \leq P \leq 1, \omega_{S}^{0}$ $\leq \omega_{S} \leq 1$; in particular $\omega_{S}=\omega_{S}^{0}$ if $P=0$ and $\omega_{S} \rightarrow 1$ as $P$ approaches 1. Also, Eq. (A1) ensures that for a given value of $P$, or degree of multiple ionization, the increase in $\omega_{S}$ becomes asymptotically insignificant when $\omega_{S}^{0} \rightarrow 1$, i.e., in heavy target elements and, especially, for the innermost shells of such atoms. On the other hand, this increase is indeed very rapid when $\omega_{S}^{0} \ll 1$; large multiple ionization is expected in the $L$ shell of relatively light target elements whose $\omega_{L}^{0}$ are small; for example, in the target range $28 \leq Z_{2} \leq 46$ of our present experiment $\omega_{L}^{0}$ ranges from 0.009 to 0.05 .
Ionization theories, which work so well for inner shells, no longer apply in calculation of $P$. We propose to calculate the probability $P$ as $P=\sigma / 8 \pi a_{0}^{2}$ where $\sigma$ is the cross section for a transfer of energy over all its possible values to "an outer-shell" electron and $8 \pi a_{0}^{2}$ is the wavemechanical cross section $4 \pi a_{0}^{2}$ (based on the outer-shell radius estimated as the Bohr radius $a_{0}$ ) doubled on the account of a pair of two electrons of opposite spin in the shell. ${ }^{28}$

Quantum and semiclassical ionization theories employ a quantum description of the electron before and after the ionization. While inner shells are well represented by screened hydrogenic wave functions, the knowledge of outer shells is less precise unless cumbersome numerical schemes are used; the outer shells, although still classified according to well-defined quantum numbers, elude quantum representation in a simple analytical form. In accord with the correspondence principle they are more suitable to a classical interpretation as states of higher principal quantum numbers. Multiple ionization of outer shells is therefore amenable to the classical description as a binary encounter between the projectile and the outer-shell electron.

The BEA consists of two steps: (1) the cross section for energy transfer to a free electron is derived, (2) the electron is reconsidered as bound and embodied with a microcanonical distribution of velocities, as defined by its binding energy, and the cross section is averaged out with this distribution. It can be proven that the BEA is equivalent to a quantum approach if the ejected electron is represented by a plane wave, ${ }^{29}$ i.e., as if the electron were completely free from the target's nucleus influence. This explains why ionization of inner shells, which expels the electron into the target's continuum, is in general poorly described by the BEA. Ionization of a weakly bound, outer-shell electron-which liberates this electron into a practically free state-is by far more suitable to this classical approach.

On the scale of projectile velocity $v_{1}$, which is larger than the Bohr velocity $v_{0}$, the outer-shell electrons move so slowly that they can be assumed to be at rest. The averaging over their velocities is no longer required after such an assumption is made. The classical formula for $d \sigma / d T$, the cross section for transfer of energy $d T$ by a projectile of charge $Z_{1}$ and velocity $v_{1}$ to a stationary electron, was introduced by Thomson some 80 years ago. ${ }^{30}$ Remnants of his otherwise defunct plum-pudding view of an atom survive if one is only looking at the atom's response in its periphery. On the scale of their spatial extention, weakly bound electrons in these outer parts of the atom experience indeed an essentially uniform potential of its nucleus.

Thus, using $d \sigma / d T$ of Thomson, ${ }^{30}$ we write ${ }^{31} P$ as

$$
P=\int_{T_{\min }}^{T_{\max }} \frac{d \sigma}{d T} d T / 8 \pi a_{0}^{2}=\frac{Z_{1}^{2}}{4 v_{1}^{2}}\left(\frac{1}{T_{\min }}-\frac{1}{T_{\max }}\right)
$$

While the maximum energy transfer $T_{\max }$ is easily found as 


$$
T_{\max }=\frac{1}{2} M_{1} v_{1}^{2}\left[4 M_{1} m_{e} /\left(M_{1}+m_{e}\right)^{2}\right]=2 m_{e} v_{1}^{2}=2 v_{1}^{2}
$$

in atomic units, $T_{\min }$-the binding energy of an outershell electron-is somewhat uncertain for lack of a unique procedure to arrive at the binding energy that would be representative of all outer-shell electrons. Henceforth, we will assume that $T_{\min }=\frac{1}{2} \beta m_{e} v_{0}^{2}$ (or $\frac{1}{2} \beta$ in atomic units) where $\beta$ is a dimensionless parameter; to the extent that outer shells of any atom resemble the ground state of the hydrogen atom we expect $\beta$ to be of order of 1 . In principle, one should differentiate amongst various outer subshells and assign to them different constants. We will, however, adopt one value of $\beta$ that represents the mean value of binding energy per electron in the outer shells. This is a common procedure in stopping-power studies where $I$, the mean binding energy defined as $I$ $=\exp \left(\sum_{S} f_{S} \ln I_{S}\right)$ with $f_{S}$ being the dipole oscillator strength, is often used; calculations of $I$ for a neutral atom can be fitted ${ }^{32}$ by $I / Z_{2}=0.6\left(1+0.7 / Z_{2}^{2 / 3}\right)$ in units of $\frac{1}{2} m_{e} v_{0}^{2}=13.6 \mathrm{eV}$. For $28 \leq Z_{2} \leq 46$, this implies $\beta=0.64$. However, to obtain a good fit to stopping-power data as well as on theoretical grounds, ${ }^{33}$ the calculated $I / Z_{2}$ is usually multiplied by $\sqrt{2}$ so that our $\beta$ would have to be set equal to 0.9 , a number indeed close to unity.

Combining Eq. (A1) with (A2) and setting the $T$ limits of integration as discussed above, our formula reads

$$
\omega_{S}=\omega_{S}^{0} /\left[1-\frac{Z_{1}^{2}}{2 \beta v_{1}^{2}}\left[1-\frac{\beta}{4 v_{1}^{2}}\right]\left(1-\omega_{S}^{0}\right)\right],
$$

where $\beta$ is left as the only adjustable parameter. This formula could be straightforwardly applied to correct the $K$ shell $x$-ray fluorescence yield for multiple ionization. The conversion of the $L$-shell ionization cross sections to x-ray production cross sections also requires the knowledge of the effect of multiple ionization on the Coster-Kronig transition rates. We extend our result, Eq. (A3), to the total $L$-shell cross-section conversion by the expedience of applying it to $\bar{\omega}_{L}^{0}$, the mean value of the fluorescence yield defined operationally as $\sigma_{L X} / \sigma_{L}$.

Restrictions on the use of Eq. (A3) follow from the physical requirement that $0 \leq P \leq 1$ in Eq. (A2). Once Eq. (A2) is conveniently rewritten as $P=x^{2} / 2 \beta-x^{4} / 8 Z_{1}^{2}$ in terms of the scaling variable $x \equiv Z_{1} / v_{1}$, it is easily seen that $P$ has a threshold at $x_{\text {th }}=2 Z_{1} / \beta^{1 / 2}$ or is nonzero for $v_{1}>\beta^{1 / 2} / 2$; with $\beta \sim 1, P$ is never zero in practice since the projectile velocities are typically larger than the Bohr velocity. It can also be instantly recognized that $P$ peaks at $x_{\max }=Z_{1}(2 / \beta)^{1 / 2}$ or at $v_{1}^{\max }=(\beta / 2)^{1 / 2}$ where it attains a value $P^{\max }=Z_{1}^{2} / 2 \beta^{2}$. This means that there is no restrictions on the use of Eq. (A3) when hydrogen $\left(Z_{1}=1\right)$ ions are employed, but for heavier $\left(Z_{1}>1\right)$ projectiles with $P<1$ only for sufficiently high velocities such that

$$
v_{1}>Z_{1}\left[1+\left(1-2 \beta^{2} / Z_{1}^{2}\right)^{1 / 2}\right]^{1 / 2} / 2 \beta^{1 / 2}
$$

or, with $\beta \cong 1$, for $v_{1} \geq Z_{1} /(2 \beta)^{1 / 2}$. Thus our method applies effectively to light projectile ions and, if $Z_{1}>1$, becomes inoperative when $Z_{1} / v_{1} \geq(2 \beta)^{1 / 2}$.
Heavy projectiles are known ${ }^{34}$ to produce a significant amount of multiple vacancies in outer shells. But these strong perturbations cannot be described by $P$ of Eq. (A2) because this probability was derived in the first order (note that $\sigma \propto Z_{1}^{2}$ ) of the scattering theory. When $Z_{1}>1$ (where 1 is the scattered electron's charge in magnitude and atomic units) the linear response theory applies only at high velocities [see the inequality (A4)] where it results in a physically meaningful $P<1$. It appears that at such velocities the outer-shell ionization probability scales with $\boldsymbol{Z}_{1} / v_{1}$ after $\boldsymbol{Z}_{1}$ is replaced with $\boldsymbol{Z}_{\text {eff }}$, an effective projectile charge in heavy-ion-atom collisions. ${ }^{35}$ The probabilities, calculated in a simplified binary-encounter model ${ }^{36}$ and in a large-impact-parameter quantum-mechanical scheme, ${ }^{37}$ are functions of $x_{\text {eff }} \equiv Z_{\text {eff }} / v_{1}$ only and are indeed in good agreement with the compiled data (see Fig. 4 in Ref. 35) when $x_{\text {eff }}<1$. Our formula, $P=x_{\text {eff }}^{2} / 2 \beta$ $-x_{\text {eff }}^{4} / 8 Z_{\text {eff }}^{2}$, gives equally good agreement in this highvelocity regime and predicts a slight additional dependence of $P$ on $Z_{\text {eff }}$. The scatter in the data ${ }^{35}$ does not allow for a definite decision on which of these calculated probabilities gives the best results.

We close this Appendix with a discussion of the role of the projectile's electrons in multiple ionizations of the target's outer shells. Based on Thomson's cross section the probability of outer-shell ionization per each electron ${ }^{38}$ on the projectile, $P_{e}$, is smaller than $P$ of Eq. (A2); since

$$
P_{e} / P=\frac{1}{Z_{1}^{2}} \frac{1-\beta / v_{1}^{2}}{1-\beta / 4 v_{1}^{2}}
$$

we estimate, with $v_{1} \geq Z_{1} /(2 \beta)^{1 / 2}$, that

$$
\frac{1-2\left(\beta / Z_{1}\right)^{2}}{1-\left(\beta / Z_{1}\right)^{2} / 2} \frac{1}{Z_{1}^{2}} \lesssim P_{e} / P \leq \frac{1}{Z_{1}^{2}} .
$$

For ${ }_{2} \mathrm{He}^{+}$and $\beta=0.87$ in our experiment this translates to $0.17 \leq P_{e} / P \leq 0.25$. Thus it appears that we have underestimated the probability for multiple ionizations by some $20 \%$ because of the neglect of an electron on the helium ion. It is tempting to change $P$ to $P+P_{e}$ according to Eq. (A5) and fit $\beta$ to our data again; a new value $\beta \cong 1$ would then be obtained.

However, two facts have to be considered. First, the straightforward addition of $P$ 's implies an incoherent addition of the squares of the transition amplitudes for multiple ionization by the electron and nucleus of the projectile. This is only an upper bound on a joint $P$ since the amplitudes differ both in sign and phase. In fact, adopting the principle that the nucleus screened by electrons should yield a smaller $P$, some kind of subtraction of $P$ 's would be more justified. The second fact is that Thomson's classical formula does not apply when two identical fermions are scattered. For electron-electron collisions, $d \sigma / d T \propto T^{-2}$ of Thomson ought to be replaced ${ }^{39}$ with

$d \sigma / d T \propto T^{-2}+\left(T_{\max }-T\right)^{-2}+2 s T^{-1}\left(T_{\max }-T\right)^{-1} \cos \Phi$

where $\Phi \equiv \ln \left[T /\left(T-T_{\max }\right)\right] / v_{1}$ and $s=+1$ or -1 for parallel or antiparallel spins of the colliding electrons. Assuming a random orientation of the spins and averag- 
ing over it, one can drop the purely quantum-mechanical exchange term and simplify this expression to $d \sigma / d T \propto T^{-2}+\left(T_{\max }-T\right)^{-2}$, the classical Rutherford cross section for scattering of identical and spinless particles. $^{40} \mathrm{~A}$ divergence of the integral in the upper limit might be interpreted as unphysical, as such divergences often appear when scattering in a Coulomb field is considered. ${ }^{41}$ In the spirit of renormalization theories, ${ }^{42}$ we do not evaluate the integral of $d \sigma / d T$ in this limit but rather renormalize the Thomson cross section-which erronously presumes unsymmetrized representation for two identical particles-to the Rutherford classical cross section at $T=T_{\min }$. This changes $P_{e}$ to $P_{e}\left[1-\left(T_{\min } / T_{\max }\right) /\left(1-T_{\min } / T_{\max }\right)\right]$ which, with $T_{\min } / T_{\max }=\beta / v_{1}^{2}$ and the $v_{1} \geq Z_{1} /(2 \beta)^{1 / 2}$ restriction, lowers the lower bound in the inequality (A6) by a factor $\left\{1-2\left(\beta / Z_{1}\right)^{2} /\left[1-2\left(\beta / Z_{1}\right)^{2}\right]\right\}^{-1}$. Thus, for ${ }_{2} \mathrm{He}^{+}$and $\beta=0.87, P_{e} / P \geq 0.17 / 2.6 \simeq 0.07$ in the low-velocity limit.

To summarize this discussion, the neglect of multiple ionizations by the electron on the helium ion leads to a $7-25 \%$ error in our estimate of the probability of multiple ionizations. This error is rather large at higher projectile velocities but the effect of multiple ionizations subsides at such velocities. A proper treatment of multiple ionization by partially stripped ions requires a cautious consideration of symmetrization of classical cross sections for electron-electron scattering and more rigorous rules for superposition of the probabilities for multiple ionization by the ion's nucleus and its electrons. An alternative approach would be to modify the target-electron-projectile-nucleus interaction for screening due to electrons on the projectile and to develop a classical theory for inelastic scattering in a non-Coulombic field. This alternative is perhaps more appealing but not without a caveat: the strict identity between quantum and classical cross sections for scattering by a fixed charge $e^{43}$ can no longer be proven when the scattering tied is not Coulombic.
${ }^{1}$ O. Benka, Nucl. Instrum. Methods B 4, 279 (1984), and references therein.

${ }^{2}$ T. L. Hardt and R. L. Watson, At. Data. Nucl. Data Tables 17, 107 (1976).

${ }^{3}$ T. J. Gray, Methods of Experimental Physics, edited by P. Richard (Academic, New York, 1980), Vol. 17, p. 193.

${ }^{4}$ P. Komarek, Acta. Phys. Austriaca 27, 369 (1968).

${ }^{5}$ K. Shima, J. Makino, and M. Sakisaka, J. Phys. Soc. Jpn. 30, 971 (1971).

${ }^{6}$ T. M. Button, R. K. Rice, J. L. Duggan, and F. D. McDaniel, IEEE Trans. Nucl. Sci. NS-26, 1139 (1979); T. M. Button, M. S. Thesis, North Texas State University, 1979.

${ }^{7}$ G. S. Khandelwal, B.-H. Choi, and E. Merzbacher, At. Data 1, 103 (1969); see B.-H. Choi, E. Merzbacher, and G. S. Khandelwal, ibid. 5, 291 (1973) for extended $L$-shell PWBA tables.

8J. H. McGuire and K. Omidvar, Phys. Rev. A 10, 182 (1974).

${ }^{9}$ See W. Brandt and G. Lapicki, Phys. Rev. A 23, 1717 (1981), for direct ionization; and G. Lapicki and F. D. McDaniel, Phys. Rev. A 22, 1896 (1980); 22, 975(E) (1981), for electroncapture calculations. The ECPSSR theory of inner-shell ionization in its latest formulation, was given in the paper of this reference. The ECPSSR cross sections (throughout the text, in all figures, and Table I) were calculated, according to the papers listed in this reference, as sums of direct-ionization and electron-capture cross sections.

${ }^{10}$ W. Brandt and G. Lapicki, Phys. Rev. A 10, 474 (1974).

${ }^{11}$ W. Brandt and G. Lapicki, Phys. Rev. A 20, 465 (1979).

${ }^{12}$ M. Poncet and C. Engelmann, Nucl. Instrum. Methods 149, 461 (1978).

${ }^{13}$ K. Ishii, K. Sera, A. Yamadera, M. Sebata, H. Arai, and S. Morita, Phys. Rev. A 25, 2511 (1982).

${ }^{14}$ A. P. Jesus, I. R. Pimental, and J. S. Lopes, Nucl. Instrum. Methods 214, 29 (1983).

15P. M. Kocur, J. L. Duggan, R. Mehta, J. Robbins, and F. D. McDaniel, IEEE Trans. Nucl. Sci. NS-30, 1580 (1983).

${ }^{16}$ R. Mehta, J. L. Duggan, J. L. Price, F. D. McDaniel, and G. Lapicki, Phys. Rev. A 26, 1883 (1982).

${ }^{17}$ J. L. Duggan, P. M. Kocur, J. L. Price, F. D. McDaniel, R. Mehta, and G. Lapicki, Phys. Rev. A 32, 2088 (1985).
18J. R. Oppenheimer, Phys. Rev. 31, 349 (1928); H. C. Brinkman and H. A. Kramers, Proc. Acad. Sci. (Amsterdam) 33, 973 (1930); V. S. Nikolaev, Zh. Eksp. Teor. Fiz. 51, 1263 (1966) [Sov. Phys.-JETP 24, 847 (1967)].

${ }^{19}$ M. O. Krause, J. Phys. Chem. Ref. Data 8, 307 (1979).

${ }^{20}$ G. Basbas, W. Brandt, and R. Laubert, Phys. Rev. A 17, 1655 (1978).

${ }^{21}$ G. D. O’Kelley, R. L. Auble, L. D. Hulett, Jr., H. J. Kim, W. T. Milner, S. Raman, O. Shahal, C. R. Vane, J. P. Young, and G. Lapicki, Nucl. Instrum. Methods B 3, 78 (1984).

${ }^{22}$ H. Paul, Nucl. Instrum. Methods B 4, 211 (1984); H. Paul and J. Muhr, Phys. Rep. 135, 47 (1986), and references therein.

${ }^{23}$ The ECPSSR curves (-. - - $)$ ) in Fig 5 are somewhat higher than the ECPSSR curves in Figs. 1 and 2 of Ref. 14 because we have included in the ECPSSR calculations (Ref. 9) the contribution of electron capture which is larger for $\alpha$ particles than for deuterons. If only direct ionization is considered, the binding effect indeed increases with the decreasing $Z_{2}$ as illustrated by the curves in Figs. 1 and 2 of Ref. 14. However when electron capture-that contributes as much as $12 \%$ to total ionization of ${ }_{32} \mathrm{Ge}$ by ${ }_{2} \mathrm{He}^{2+}$-is added, $\sigma_{L X}\left({ }_{2}^{4} \mathrm{He}^{2+}\right) / 4 \sigma_{L X}\left({ }_{1}^{2} \mathrm{H}^{+}\right)$becomes, in agreement with the experimental ratios (Ref. 14), larger for smaller $Z_{2}$. Yet, as Fig. 5 shows, these ratios are still underestimated by the ECPSSR theory if the identical fluorescence yields are used in the aftermath of $\alpha$ particle and deuteron bombardment.

${ }^{24}$ R. Gundersen, J. M. Hansteen, and L. Kocbach, Nucl. Instrum. Methods 192, 63 (1982); J. S. Lopes, A. P. Jesus, and M. F. daSilva, J. Phys. B 15, 1749 (1982); IEEE Trans. Nucl. Sci. NS-30, 954 (1983). Based on more recent semiclassical calculations L. Kocbach, Nucl. Instrum. Methods B 4, 248 (1984), and G. Mehler, T. de Reus, U. Müller, J. Reinhardt, B. Müller, W. Grieiner, and G. Soff, Nucl. Instrum. Methods A 240, 559 (1985), assert, however, that the ECPSSR theory underestimates the binding effect.

${ }^{25}$ A. P. Jesus, J. S. Lopes, and J. P. Riberio, J. Phys. B 18, 2453 (1985).

${ }^{26}$ Although $P$ 's in Eq. (A2) are identical for all target atoms and a given projectile, $\omega_{S}^{0}$ for heavy elements are corrected by 
Eq. (A3) to a lesser degree because of the $1-\omega_{S}^{0}$ factor which is smaller for larger $Z_{2}$. Corrections that are for gold and lead in Ref. 25 are indeed smaller than for light elements in Ref. 14. However, Eq. (A3) still does not fully explain the observed (Ref. 25) discrepancy for $L$-shell ionization in uranium.

${ }^{27}$ An approach which lumps all outer shells into a single entity is consistent with the quasiparticle approximation in photoelectron spectroscopy that treats the vacancy decay as a collective phenomenon. See A. S. Kheifets, M. Y. Amusia, and V. G. Yarzhemsky, J. Phys. B 18, L343 (1985), on the validity of the quasiparticle approximation.

${ }^{28}$ See N. F. Mott and H. S. W. Massey, The Theory of Atomic Collisions, 3rd ed. (Clarendon, Oxford, 1965), Chaps. II.6 and XII.1, for genesis of $4 \pi a_{0}^{2}$ as the maximum cross section in scattering of spinless particles. The ionization probability per electron in the manifold of outer states is calculated as $\sigma / 8 \pi a_{0}^{2}$ because there are twice as many electrons in it than for the case in which the spin is ignored.

${ }^{29}$ D. H. Madison and E. Merzbacher, in Atomic Inner-Shell Processes, edited by B. Crasemann (Academic, New York, 1975), Vol. I. p. 1. M. Gryziński and J. A. Kunc, J. Phys. B 19, 2479 (1986), note that greater success of the standard BEA in treatment of inner-shell ionization is due to inadequacies of the single-electron description of outer shells. We argue-not in contradiction to this observation-that classical theories of ionization are more justified for outer shells once these theories are extended beyond the single-electron approximation.

30J. J. Thomson, Philos. Mag. 23, 449 (1912). The Thomson cross section as a classical treatment of ionization is reviewed in Chaps. 3 of N. Bohr, K. Dan. Vidensk. Selsk. Mat.-Fys. Medd. 18, No. 8 (1948) and of M. R. C. McDowell and J. P. Coleman, Introduction to the Theory of Ion-Atom Collisions (North-Holland, Amsterdam, 1970). Bohr traces the Thomson formula to J. J. Thomson in Conduction of Electricity through Gases, 2nd ed. (Cambridge University Press, Cambridge, 1906). Although Thomson's considerations of ionization-especially by then so-called Becquerel rays in the 1903 first edition of this book (see pp. 344 and 345)-give a qualitative discussion of this phenomenon, these books do not state explicitly the formula that is derived in Thomson's 1912 article.

${ }^{31}$ In the atomic units, $m_{e}=1$, the charge of the projectile $Z_{1} e=Z_{1}$, and its velocity $v_{1}$ is expressed in $v_{0}$ which is not written explicitly (the Bohr velocity $v_{0}=1$ in the atomic units).

${ }^{32}$ W. Brandt, Health Phys. 1, 11 (1958). These calculations were based on a statistical description of an atom and tailored to the definition of $I, \ln I=\Sigma_{S} f_{S} \ln I_{S}$, as required in stoppingpower formulas. A value of $I$ should instead be derived from $I^{-1}=\sum_{S} f_{S} I_{S}^{-1}$ in which, as demanded by Eq. (A2), the sum ought to be only over the ionization energies $I_{S}$ for outer shells and $f_{S}$ would denote the fractional occupancy of these shells; we have not performed such a calculation.

33J. Lindhard and M. Scharff, K. Dan. Vidensk. Selsk. Mat.Fys. Medd. 27, No. 15 (1953), argue that atoms respond with a restoring force which is proportional to the sum of the squares of two frequencies-the orbital and plasma frequencies-and prove in a statistical model of an atom that these frequencies are the same; hence the multiplicative factor $\sqrt{2}$ follows. The crucial assumption about a plasmalike response of the atom applies particularly well to its outer shells and is consistent with a view of their multiple ionization as a collective response to the perturbing projectile.

${ }^{34}$ D. Schneider, M. Prost, R. DuBois, and N. Stolterfoht, Phys. Rev. A 25, 3102 (1982); J. A. Tanis, S. M. Shafroth, W. W. Jacobs, T. McAbee, and G. Lapicki, ibid. 31, 750 (1985); W. Uchai, G. Lapicki, W. T. Milner, S. Raman, P. V. Rao, and C. R. Vane, J. Phys. B 18, L389 (1985).

${ }^{35}$ I. Kádár, S. Ricz, V. A. Shchegolev, B. Sulik, D. Varga, J. Vegh, D. Berényi, and G. Hock, J. Phys. B 18, 275 (1985).

${ }^{36}$ B. Sulik, G. Hock, and D. Berényi, J. Phys. B 17, 3239 (1984).

${ }^{37}$ L. Végh, Phys. Rev. A 32, 199 (1985).

${ }^{38} P_{e}$ is calculated as $P$ of Eq. (A2) with $Z_{1}$ replaced by -1 and $T_{\max }=v_{1}^{2} / 2$ instead of $T_{\max }=2 v_{1}^{2}$.

${ }^{39}$ See N. F. Mott and H. S. W. Massey, Ref. 28, Chap. XI.5, Eq. (26).

${ }^{40}$ E. Rutherford, J. Chadwick, and C. D. Ellis, Radiations from Radioactive Substances (Cambridge University Press, Cambridge, 1930), p. 262; also N. F. Mott and H. S. W. Massey, Chap. XI.4 of Ref. 28.

41J. M. Jauch and F. Rohrlich, The Theory of Photons and Electrons, 2nd ed. (Springer, New York, 1976), see Supplement S4.

${ }^{42}$ Note that $T=T_{\max }$ corresponds to the zero impact parameter at which the closest distance between the colliding electrons is $2 / v_{1}^{2}$. This distance is typically smaller and at most-when $v_{1} \cong Z_{1} /(2 \beta)^{1 / 2}$ and $Z_{1}=2$-comparable to the de Broglie wavelength, $1 / v_{1}$, of the projectile's electron. In such collisions the classical picture breaks down, see Eq. (1.3.9) of Bohr in Ref. 30; the divergence at $T=T_{\max }$ arises outside the realm of classical physics, perhaps as a consequence of the premature elimination of the quantum-mechanical exchange term.

${ }^{43}$ See N. F. Mott and H. S. W. Massey, Ref. 28, p. 53. 